\title{
Statistical Approach Optimizing Slant Feed Grinding
}

\author{
Keita SHIMADA ${ }^{1 \mathrm{a}}$, Pay Jun LIEW ${ }^{1 \mathrm{~b}}$, Tianfeng ZHOU ${ }^{1 \mathrm{c}}$, Jiwang YAN ${ }^{1 \mathrm{~d}}$, and Tsunemoto KURIYAGAWA ${ }^{\text {le }}$ \\ ${ }^{1}$ Department of Mechanical Systems and Design, Graduate School of Engineering, Tohoku University, \\ Aoba 6-6-01, Aramaki, Aoba-ku, Sendai, 980-8579, Japan, \\ a shimada@pm.mech.tohoku.ac.jp ${ }^{\mathrm{b}}$ payjun@pm.mech.tohoku.ac.jp ${ }^{\mathrm{c}}$ zhoutf@pm.mech.tohoku.ac.jp \\ dyanjw@pm.mech.tohoku.ac.jp ${ }^{\mathrm{e}}$ tkuri@m.tohoku.ac.jp
}

\begin{abstract}
:
Micro-grinding is one of the methods to create submillimeter-structures, and is required to improve ground surface roughness. Slant feed grinding is a machining method to improve ground surface roughness, but the improving mechanism has not been clarified yet. This paper proposed a theoretical method for calculating ground surface roughness of slant feed grinding using a statistical approach. A self-developed micro-grinding device was then employed to confirm the calculation method experimentally.
\end{abstract}

Keywords: Slant feed grinding, Statistical calculation, Ground surface roughness, Micro-grinding

\section{Introduction}

Micro-machining techniques have been recently required to manufacture sophisticated products at microscale. Micro-grinding, which refers to a grinding method using wheels of less than one millimeter in diameter, is one of submillimeter structures processing methods. Compared with other typical micro-machining methods, such as electrical discharge machining (EDM) and ultrasonic machining (USM), micro-grinding has some advantages including applicability for various kinds of materials and forming ability to fabricate complicated three-dimensional geometrical structures. However, due to using small diameter wheels for micro-grinding, wheel bending occurs easily. In addition, due to lower abrasive grain distribution on the wheels in micro-grinding than that on the wheels in conventional grinding, ground surface roughness in micro-grinding gets worse. To solve these problems, we have been applied vibration assisted grinding, including ultrasonic-assisted grinding and oscillating grinding, to micro-grinding [1-2]. Vibration assisted grinding has some advantages, including improving ground surface roughness and reducing grinding force, which have been reported by authors such as Kumabe et al., Nomura et al., Tanaka et al., Unno et al., Nakagawa et al., and Yasui et al. [3-8]. However, the mechanism of improving ground surface roughness by vibration assisted grinding has not been completely clarified yet.

Theoretical methods for calculating ground surface roughness of conventional grinding have been proposed by authors such as Sato et al., Ono et al, Orioka et al and Matsui et al. [9-15], especially the model of Matsui et al. can easily handle cutting edge distribution and its randomness. Therefore, this paper proposes a theoretical model that was modified from the model proposed by Matui et al. was utilized for calculating ground surface roughness of slant feed grinding, as the first step of clarification of ground surface improving mechanism of vibration grinding. The calculated results were then confirmed by experiments using a self-developed ultrasonic-assisted micro-grinding device.

\section{Theoretical Method for Calculating Ground Surface Roughness of Slant Feed Grinding}

\subsection{Categorization of Vibration Assisted Grinding}

Vibration assisted grinding is divided into two sections based on the vibration frequency, and generally, the low and high frequency vibration assisted grinding are categorized as oscillating grinding and ultrasonic-assisted grinding, respectively. To define the two sections more strictly, we have defined them based on the velocity ratio, $n=2 \pi f A / V$ in this research, where $f, A$ and $V$ are vibration frequency, half amplitude of vibration and wheel rotational velocity, respectively. When $n<1$, the vibration assisted grinding belongs to the oscillating grinding, otherwise it belongs to ultrasonic-assisted grinding. Oscillating grinding includes slant feed grinding as $n=0$, and this paper proposes a theoretical method to calculate ground surface roughness of slant feed grinding.

\subsection{Theoretical Assumptions and Model}

The theoretical method of Matsui et al. is suitable for calculating ground surface roughness, but that can only apply to conventional grinding. Therefore in this paper, an attempt has been made to modify the theoretical method for calculating slant feed grinding. As the basis of theoretical model, theoretical assumptions were declared as follows [12-15].

(1) Every cutting edge is a circular cone with a constant tip angle $2 \alpha$ and the conical axis is in the radial direction of the grinding wheel.

(2) Cutting edges are distributed "uniformly at random" on the surface of a grinding wheel. The mean volume occupied by a grain tip takes constant $W_{0}$ in any place of the wheel if the space is sufficiently large.

(3) Shapes of scratches on the ground surface are the same as those of grain tips. The elastic deformations of both the workpiece and the cutting edges are neglected.

Figure 1 shows a theoretical model of slant feed grinding including the definition of coordinate systems of 


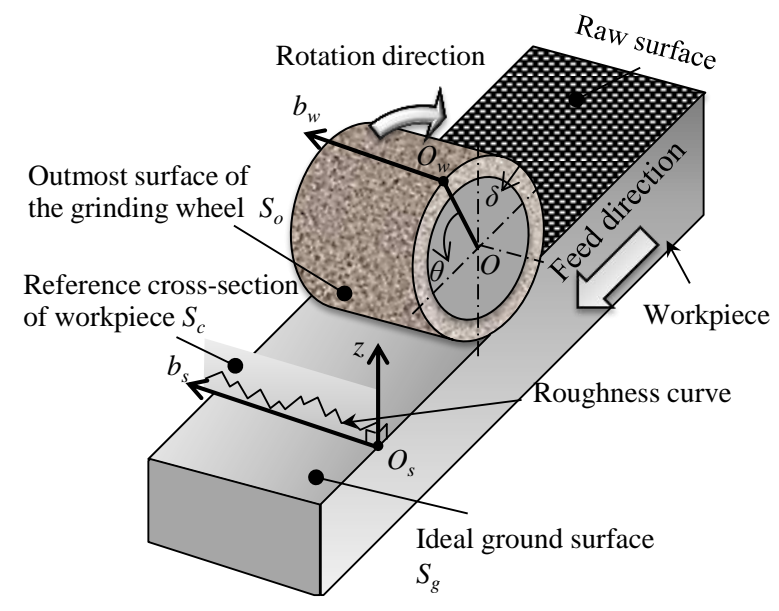

Figure 1: Schematic of slant feed grinding. The coordinate system $\Sigma_{w}\left(O_{w}, \delta, \theta, b_{w}\right)$ is fixed on the grinding wheel. The coordinate system $\Sigma_{s}\left(O_{s}, z, b_{s}\right)$ is fixed on the ideal ground surface $S_{g}$.

the grinding wheel $\Sigma_{w}$ and the ground surface $\Sigma_{s}$. The outmost surface of the grinding wheel $S_{o}$ is defined as the cylindrical surface containing the farthest cutting tip from the grinding wheel axis. $\Sigma_{w}$-origin $O_{w}$ is fixed anywhere on $S_{o}$. Any point in the wheel, $P_{w}$, can be determined by the radial distance $\delta$, the azimuth $\theta$ and the breadth $b_{w}: \delta$ is the distance from $S_{o}$ to $P_{w} ; \theta$ is the angle from the plane containing the wheel axis and $O_{w}$ to the plane containing the wheel axis and $P_{w} ; b_{w}$ is distance from $O_{w}$ to the foot of perpendicular from $P_{w}$ on $S_{o} . \Sigma_{s}$ is fixed on the ideal ground surface $S_{g}$, which comes into contact with $S_{o}$. $\Sigma_{s}$-origin $O_{s}$ is fixed anywhere on $S_{g}$. Any point in the reference cross-section of workpiece $S_{c}, P_{s}$, can be determined by the height $z$ and the breadth $b_{s}: z$ is the distance from $S_{g}$ to $P_{s} ; b_{s}$ is the distance from $O_{s}$ to the foot of the perpendicular from $P_{s}$ on $S_{g}$. Additionally the time reference $(t=0)$ is defined when $b_{s}$ and $b_{w}$ meet, and $b_{w}$ is included in $S_{c}$ when $t=\tau$. $\Sigma_{w}$ can define all positions of tip points of cutting edges on the grinding wheel, and $\Sigma_{s}$ can define all positions of the tip points of cut traces on the reference cross-section of workpiece $S_{c}$. Figure 2 shows cutting process of one cutting edge $G_{i}\left(\delta_{i}, \theta_{i}, b_{w i}\right)$. As shown in Figure 2, $G_{i}$ can cut the workpiece multiple times under certain conditions. $G_{i}$ creates its lowest cut trace during $-\pi D / V<t<\pi D / V$, and the tip point of the lowest cut trace is defined as $G_{i, 0}^{\prime}\left(z_{i, 0}, b_{s i, 0}\right)$ on $S_{c} . G_{i, j}^{\prime}\left(z_{i, j}, b_{s i, j}\right)$ is the position of the tip point of cut traces created by $G_{i}$ after $j$ wheel revolutions (when $j<0$, before $|j|$ wheel revolutions) since the lowest cut trace is created. $G_{i, j}$ is given as follows:

$\left\{\begin{array}{c}z_{i j} \\ b_{s i j}\end{array}\right\}=\left\{\begin{array}{c}\delta_{i}+h\left(\theta_{i}+2 \pi j\right) \\ b_{w i}+v_{a} \frac{D\left(\theta_{i}+2 \pi j\right)}{2 V}\end{array}\right\}$,

where $h\left(\theta_{i}\right)$ is given by a geometrical calculation as follows:
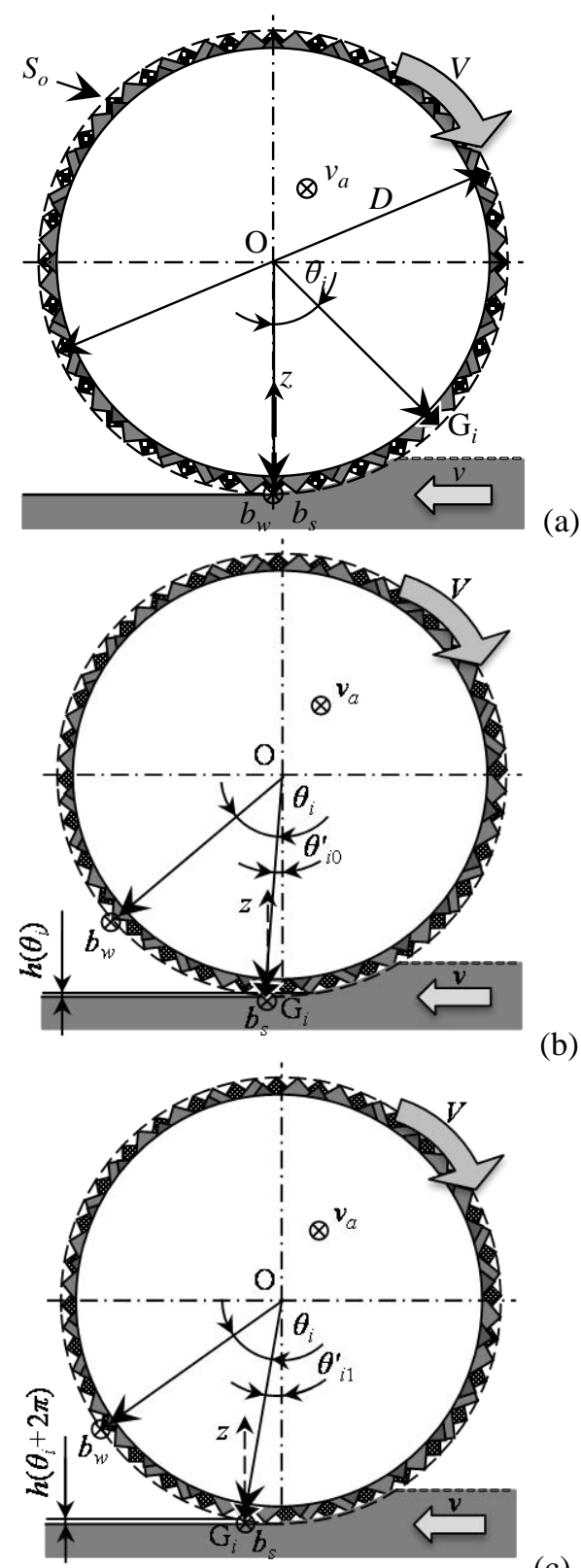

(b)

(c)

Figure 2: The position of a cutting edge on the cross-section of workpiece $S_{c}$, where $D$ is the diameter of the grinding wheel, $v$ is the workpiece feed speed, $v_{a}$ is the movement speed to axial direction and $V$ is the wheel rotational speed. (a) $t=0$, Axis $b_{w}$ and Axis $b_{s}$ coincide, (b) $t=\tau$, the tip point of an arbitrary cutting edge $G_{i}\left(\delta_{i}, \theta_{i}, b_{w i}\right)$ is included in $S_{c}$, (c) $t=\tau+\pi D / V, G_{i}\left(\delta_{i}\right.$, $\left.\theta_{i}, b_{w i}\right)$ is included in $S_{c}$ again.

$h\left(\theta_{i}\right)=\frac{D}{4}\left(\frac{v}{V}\right)^{2} \theta_{i}^{2}$,

Using Equation (1) and (2), all positions of tip points $\left\{G_{i, j}^{\prime}\right\}_{i=1,2, \ldots, j=0, \pm 1, \pm 2, \ldots}$ are determined, but note that not all cut traces can actually remain on the ground surface. Figure 3 shows a schematic of interference of cut traces among each other. As shown in Figure 3 (a), the cut trace of $G_{2,0}^{\prime}$ contains $\left\{G_{2, j}^{\prime}\right\}_{j= \pm 1, \pm 2, \ldots}$, the tip points of the other 
cut traces created by $G_{2}$. This means that the cut trace of $G_{2,0}$ removes the cut traces of $\left\{G_{2, j}^{\prime}\right\}_{j= \pm 1, \pm 2, \ldots}$ and only the cut trace of $G_{2,0}$ have the possibility to remain on $S_{c}$. Therefore, cut traces of $\left\{G_{i, 0}^{,}\right\}_{i=1,2, \ldots}$ are only effective to create ground surface in conventional grinding. Note that even the lowest cut traces cannot probably remain on $S_{c}$, shown as $G_{6,0}$. Meanwhile, as shown in Figure 3 (b), the cut traces of $\left\{G_{i, j}^{,}\right\}_{i=1,2, \ldots, j=0, \pm 1, \pm 2, \ldots}$ can remain on $S_{c}$ in slant feed grinding. These facts contribute to improving ground surface roughness. Then, the improving effect is calculated by a statistical approach.

\subsection{Calculating Ground Surface Roughness of Slant Feed Grinding}

To obtain ground surface roughness, $f_{s}\left(z, b_{s}\right)$, which is a function of tip point density on $S_{c}$, is calculated. To define the calculation interval, it is assumed that $G_{i, j}^{\prime}$ which is included in the cut traces of $\left\{G_{i, k}^{,}\right\}_{k \neq j}$ is neglected. Under this assumption, only the cut traces of $\left\{G_{i, 0}^{,}\right\}_{i=0,1,2, \ldots}$ need to be considered for calculating $f_{s}$ of conventional grinding. On the other hand, slant feed grinding has the possibility of cut traces of $\left\{G_{i, 0}^{\prime}\right\}_{i=0,1,2, \ldots}$ remaining on $S_{c}$. Figure 4 shows an example of effective and ineffective cut traces. $\left\{G_{i, j}\right\}_{j=-2,-1,0,1}$ are not included in any other cut traces, thus these tip points are effective. Meanwhile, $G_{i, 2}$ is included in the cut trace of $G_{i, 1}^{\prime}$, thus $G_{i, 2}^{\prime}$ is ineffective. Considering the geometrical relationship among each cut trace created by the same cutting edge, the maximum height tip point having the possibility to remain $G_{\max , N}\left(z_{\max , N}, b_{s \max , N}\right)$ satisfies the following equation:

$z_{\max , N}-z_{\max , N-1}=\left(b_{s \max , N}-b_{s \max , N-1}\right) \cot \alpha$,

then, using Equation (1), (2), the following solution is obtained:

$\theta_{\max }=\pi+\varphi$

where, $\varphi=\frac{v_{a} V}{v^{2}} \cot \alpha$,

consequently, the calculation interval $I(\varphi)$ is given as follows:

$I(\varphi)=[-\pi-\varphi, \pi+\varphi]$

Using Equation (1), (2), (5) and tip point density on the grinding wheel $f_{w}\left(\delta, \theta, b_{w}\right), f_{s}\left(z, b_{s}\right)$ can be obtained as follows:

$$
f_{s}\left(z, b_{s}\right)=\int_{\theta \in I(\varphi)} f_{w}\left(z-h(\theta), \theta, b_{s}-\frac{v_{a} D \theta}{2 V}\right) \frac{D}{2} d \theta,
$$

based on Assumption (2), $f_{w}\left(\delta, \theta, b_{w}\right)$ is defined as $f_{w}(\delta, \theta$, $\left.b_{w}\right) \equiv 1 / W_{0}$ (constant). By substituting $f_{w}\left(\delta, \theta, b_{w}\right)$ into Equation $(6), f_{s}\left(z, b_{s}\right)$ is obtained as follows:

$$
f_{s}(z)=\left\{\begin{array}{cc}
\frac{\pi D}{W_{0}} \sqrt{\frac{z}{h(\pi)}} & z<h(\pi+\varphi) \\
\frac{(\pi+\varphi) D}{W_{0}} & z \geq h(\pi+\varphi)
\end{array},\right.
$$

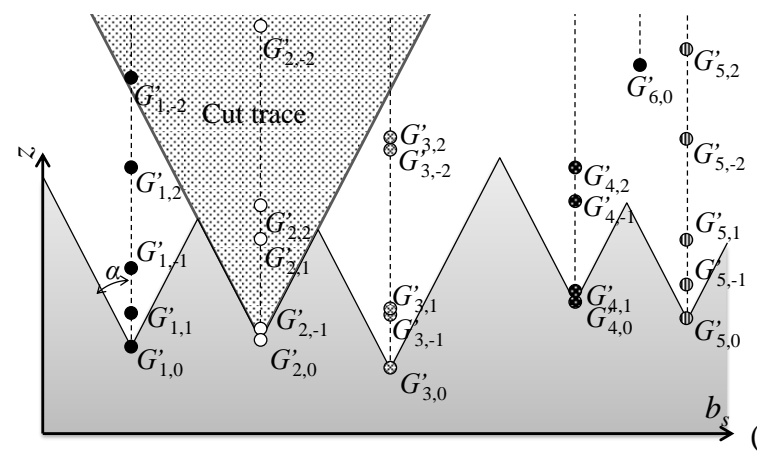

(a)

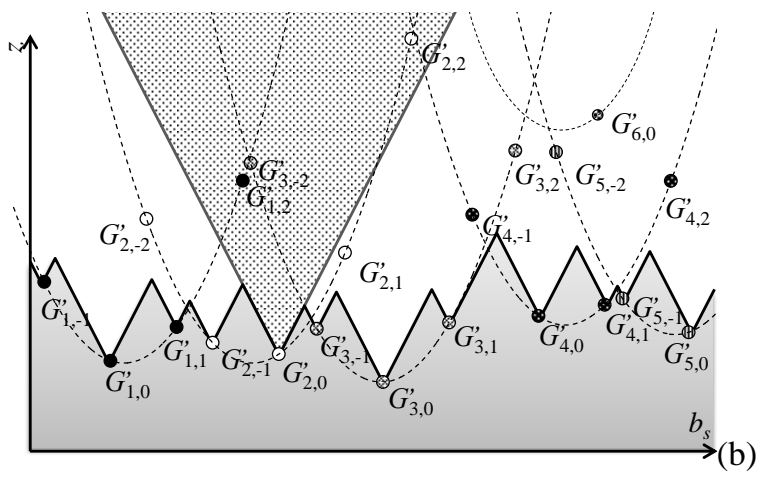

Figure 3: Schematic of the reference cross-section and tip points of cut traces. (a) The cross-section created by conventional grinding. A group of tip points of cut traces created by the same cutting edge $\left\{G_{i, j}^{\prime}\right\}_{j= \pm 1, \pm 2, \ldots}$ forms a line parallel to Axis $z$, (b) The reference cross-section created by slant feed grinding. A group of tip points of cut traces created by the same cutting edge $\left\{G_{i, j}^{\prime}\right\}_{j= \pm 1, \pm 2, \ldots}$ forms a parabola.

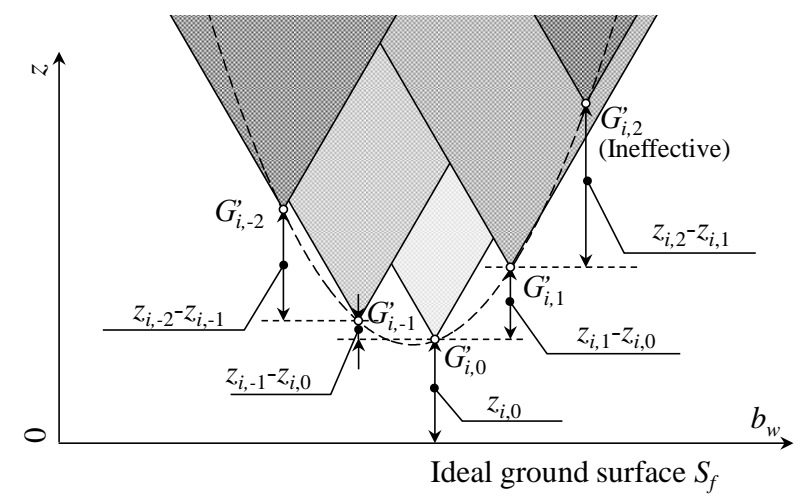

Figure 4: Positions of tip points of cut traces created by one cutting edge in slant feed grinding.

as shown in Equation (7), $f_{s}\left(z, b_{s}\right)$ is independent from the parameter $b_{s}$.

Figure 5 shows a schematic of the reference cross-section and three arbitrary points $X_{1}, X_{2}$, and $X_{3}$ are included in the area. $T\left(X_{i}\right)$ is the triangle with the vertex angle of $2 \alpha$ below Point $X_{i}$. When $T\left(X_{i}\right)$ does not include any of the tip points of cut traces, $X_{i}$ remains in the workpiece as shown $X_{1}$ and $X_{3}$; otherwise $X_{i}$ is removed from the workpiece as shown $X_{2}$. Therefore, the probability of Point $X_{i}$ remaining in the cross-section can be calculated by considering the probability of $T\left(X_{i}\right)$ containing the tip points of cut traces. $1 / f_{s}(z)$ is the mean length of interval between tip points of cut traces in height 
$z$, and the maximum interval length is assumed as $\bar{n} / f_{s}(z)$, where $\bar{n}$ is a random coefficient [16], and the probability of a tip point contained at a height of $\zeta$ in $T\left(X_{i}\right)$, $p(\zeta)$ is given as follows:

$p(\zeta)=\frac{2(z-\zeta) \tan \alpha}{\bar{n} / f_{s}(\zeta)} \quad(0 \leq \zeta \leq z)$.

$P\left(X_{i}\right)$, which is the probability of a tip point contained in $T\left(X_{i}\right)$, is calculated as summation of $p(\zeta)$. In this case, $z$ out of $X_{i}$ is the only variable for $f_{s}$, and $P\left(X_{i}\right)$ can be treated as a one-variable function $P(z)$, and given as follows:

$$
\begin{aligned}
& P\left(X_{i}\right)=P(z)=\int_{0}^{z} p(\zeta) d \zeta \\
& \frac{8}{15} \frac{z^{\frac{5}{2}}}{\left(R_{\max }^{*}(\varphi)\right)^{2} \sqrt{h(\pi+\varphi)}} \\
& z \begin{array}{c}
z<h(\pi+\varphi) \\
\frac{1}{\left(R_{\max }^{*}(\varphi)\right)^{2}}\left(z^{2}-\frac{2}{3} h(\pi+\varphi) z+\frac{1}{5}(h(\pi+\varphi))^{2}\right) \\
z \geq h(\pi+\varphi)
\end{array},
\end{aligned}
$$

where $R_{\max }^{*}(\varphi)$ is defined as follows:

$R_{\max }^{*}(\varphi)=\sqrt{\frac{\bar{n} W_{0}}{(\pi+\varphi) D} \cot \alpha}$,

and the calculating method for $R_{\max }^{*}(\varphi)$ is explained afterward.

Equation (9) also describes the Abbott-Firestone curve of ground surface. When $P(z)=1, T\left(X_{i}\right)$ must contain at least one tip point of a cut trace, and the $z$ satisfying the equation is equal to ground surface roughness in maximum height $R_{\max }(\varphi)$. Consequently, $R_{\max }(\varphi)$ is obtained by solving $P(z)=1$, and given as follows:

$$
=\left\{\begin{array}{c}
\left(\frac{15}{8}\left(R_{\max }^{*}(\varphi)\right)^{2} \sqrt{h(\pi+\varphi)}\right)^{\frac{2}{5}} \\
h(\pi+\varphi)>\sqrt{\frac{15}{8}} R_{\max }^{*}(\varphi) \\
\frac{1}{3} h(\pi+\varphi)+\sqrt{\left(R_{\max }^{*}(\varphi)\right)^{2}-\frac{4}{45}(h(\pi+\varphi))^{2}} \\
h(\pi+\varphi) \leq \sqrt{\frac{15}{8}} R_{\max }^{*}(\varphi)
\end{array} .\right.
$$

$R_{\max }^{*}(\varphi)$ is the solution of $P(z)=1$ when substituting $v=0$ into Equation (7), and especially when $\varphi=0$, it is ground surface roughness after spark-out grinding. In addition, when $\varphi=0, R_{\max }(0)$ corresponds with the equation proposed by Matsui et al. This fact means that Equation (11) can apply to ground surface roughness of conventional plunge grinding and slant feed grinding seamlessly.

Figure 6 shows the calculation results of surface

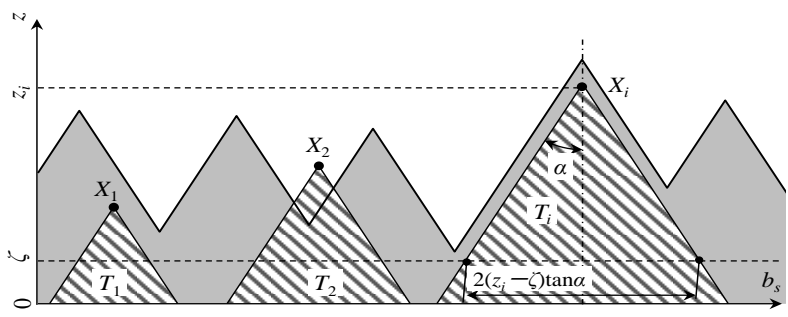

Figure 5: Schematic of the reference cross-section of workpiece.

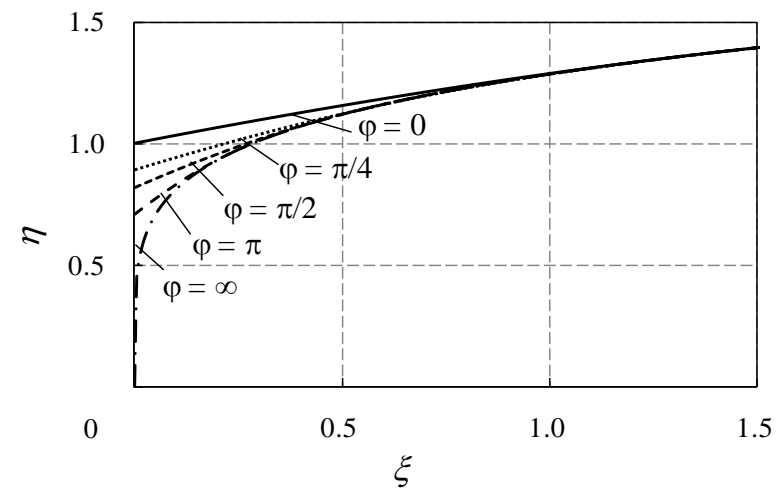

(a)Relationship between $\xi$ and $\eta$

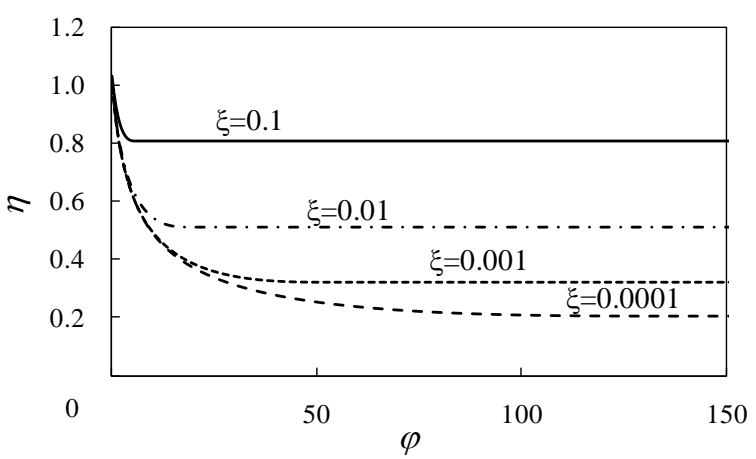

(b) Relationship between $\varphi$ and $\eta$

Figure 6: Calculation results of ground surface roughness slant feed grinding. $\xi, \eta$ and $\varphi$ are the standardized dimensionless parameters of the feed speed, the ground surface roughness in maximum height and the movement speed to axial direction, respectively.

roughness of slant feed grinding using Equation (11). Two dimensionless numbers, $\xi$ and $\eta$, are defined as follows:

$\xi \equiv \frac{h(\pi)}{R_{\max }^{*}(0)}$,

$\eta \equiv \frac{R_{\max }(\varphi)}{R_{\max }^{*}(0)}$

As shown in Figure 6 (a), when $\xi>1$, slant feed grinding has no effect on improving ground surface roughness, but otherwise slant feed grinding becomes more effective when $\xi$ is set lower. Additionally, as shown in Figure 6 (b), increasing $\varphi$ is also effective, but the improving effect has thresholds decided by $\xi$.

The mechanism of improving ground surface roughness using slant feed grinding was shown 

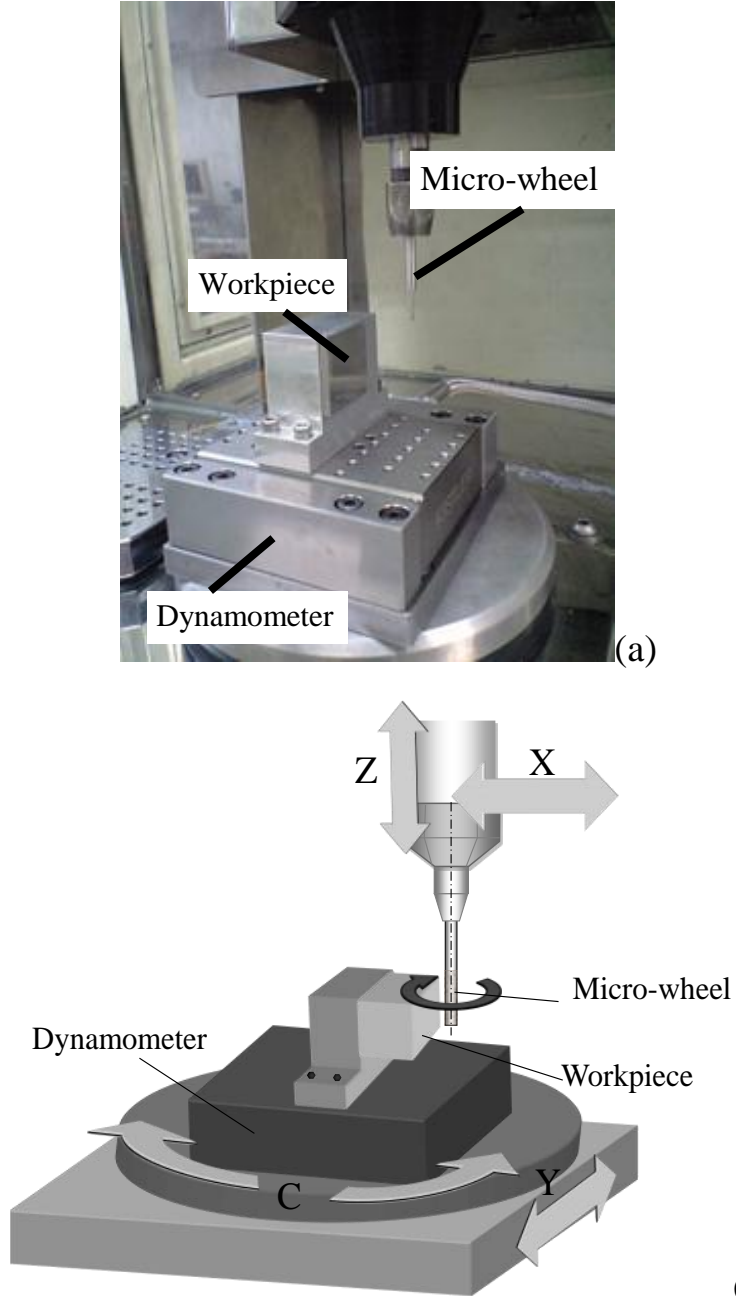

(b)

Figure 7: Experimental setup (a) Photograph of the experimental setup (b) Schematic of micro-grinding device

theoretically as above. By using this calculation, conditions of slant feed grinding can be optimized. To confirm the theoretical calculation, slant feed grinding experiments were then conducted.

\section{Slant Feed Grinding Experiments}

\subsection{Experimental Setup and Conditions}

In order to conduct vibration assisted micro-grinding, a grinding device was developed. Figure 7 shows the developed micro-grinding device and its schematic. It can perform both ultrasonic-assisted grinding and slant feed grinding/oscillating grinding by an ultrasonic transducer and computer numerical control. Slant feed grinding can be conducted by cooperative control of $\mathrm{Y}$ axis and $\mathrm{Z}$ axis. The least command increment of each axis is $0.1 \mu \mathrm{m}$ in $\mathrm{X}$, $\mathrm{Y}$ and $\mathrm{Z}$ axis, and 0.01 degree in $\mathrm{C}$ axis. The dynamometer was used to detect the contact between the grinding wheel and the workpiece. Table 1 shows the detail of experimental conditions. Grit size, Feed speed and Movement speed to axial direction were set to the shown conditions to vary the parameters $\xi$ and $\varphi$, both of which were defined in $\S 2.3$, to confirm the theoretical method.
Table 1: Slant feed grinding experimental conditions

\begin{tabular}{l|l}
\hline Workpiece & $\begin{array}{l}\text { Zirconia block } \\
13 \times 14 \times 17 \mathrm{~mm}^{3}\end{array}$ \\
\hline Grinding wheel & $\begin{array}{l}\varnothing 1 \text { electroplated mounted } \\
\text { wheel }\end{array}$ \\
\hline Abrasive grain material & Diamond \\
\hline $\begin{array}{l}\text { Grit size \# } \\
\text { (Mean diameter of grains) }\end{array}$ & $\begin{array}{l}\# 1000, \# 1500, \# 3000 \\
(15,10,6 \mu \mathrm{m})\end{array}$ \\
\hline Feed speed $v$ & $1-10 \mathrm{~mm} / \mathrm{min}$ \\
\hline Wheel rotation speed $V$ & $104 \mathrm{~mm} / \mathrm{s}(2000 \mathrm{rpm})$ \\
\hline Wheel depth of cut $\Delta$ & $50 \mu \mathrm{m}$ \\
\hline $\begin{array}{l}\text { Movement speed to axial } \\
\text { direction } v_{a}\end{array}$ & $0-6 \mathrm{~mm} / \mathrm{min}$ \\
\hline
\end{tabular}

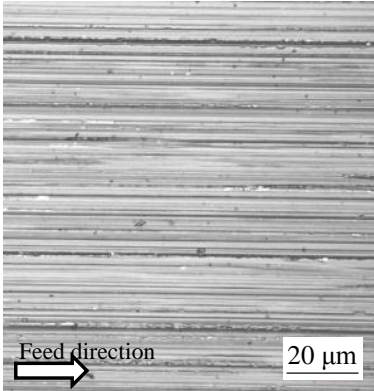

(a)

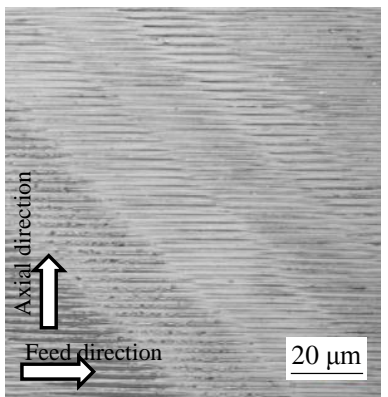

(b)
Figure 8: Photographs of ground surfaces observed with a confocal laser microscope. (a) surface created by conventional grinding (\#3000, $v=5 \mathrm{~mm} / \mathrm{min}$ ), (b) surface created by slant feed grinding (\#3000, $v=5$ $\left.\mathrm{mm} / \mathrm{min}, v_{a}=5 \mathrm{~mm} / \mathrm{min}\right)$.

\subsection{Experimental Results}

Figure 8 shows photographs of ground surfaces created by conventional grinding and slant feed grinding. The cut traces of conventional grinding was parallel to the feed direction, meanwhile the cut traces of slant feed grinding were shifted upward due to the movement to axial direction. This shows that one cutting edge cut the workpiece multiple times as shown in Figure 3.

Figure 9 shows comparison of ground surface roughness of each condition. In this study, to perform theoretical calculations, values of $\bar{n} W_{0}$, which were used in Equation (10), were determined by using the experimental results of conventional grinding for each grinding wheel. Each ground surface roughness was measured along the axial direction. As shown in Figure 9, slant feed grinding improved ground surface roughness, and improving effect thresholds can be observed depending on the grinding conditions. These results were in accord with the theoretical calculations. Therefore, this calculation method can be used to determine conditions of slant feed grinding. As an example, it could be expected that $v_{a}=3 \mathrm{~mm} / \mathrm{min}$ was enough to obtain the maximum improving effect of slant feed grinding in the case of the experiment with \#1000 grinding wheel.

\section{Conclusion}

In this paper, theoretical method for calculating ground surface roughness of slant feed grinding was proposed, 


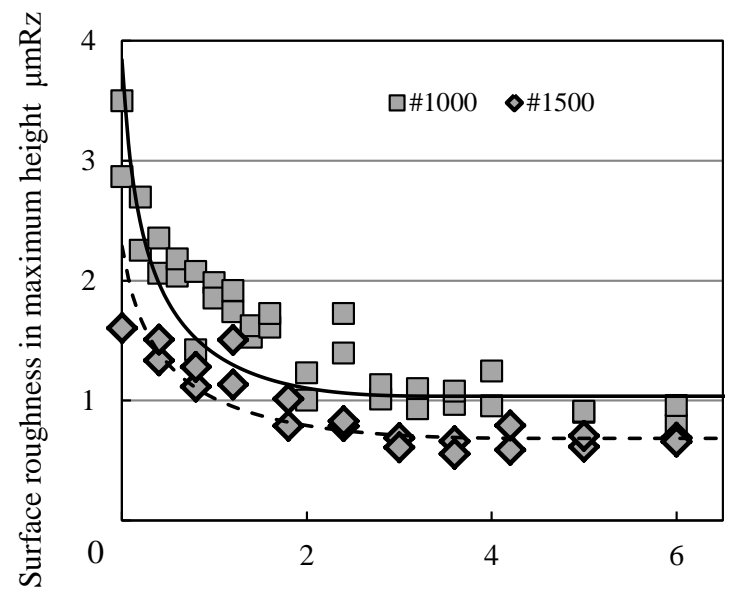

Movement speed to axial direction $\mathrm{mm} / \mathrm{min}$

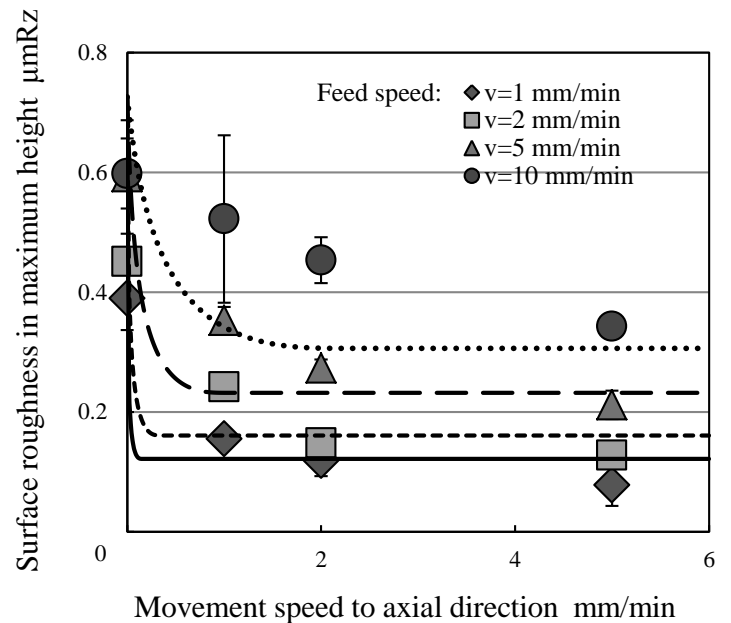

(b)

Figure 9: Comparison of experimental results and theoretical calculations of ground surface roughness. Curves indicated theoretical calculations. (a) Results of $\# 1000$ and \#1500 grinding wheel, $v=5 \mathrm{~mm} / \mathrm{min}$. (b) Results of each feed speed condition with \#3000 grinding wheel.

and the method includes the previous theoretical method proposed by Matsui et al. This calculation method was confirmed experimentally, and therefore it can be used to optimize conditions of slant feed grinding.

\section{Acknowledgement}

This study was supported by Grant-in-Aid for JSPS Fellows KAKENHI K0222850.

\section{References}

[1] Shimada, K., Tateishi, T., Yoshihara, N., Yan, J. and Kuriyagawa, T., 2009, Ultrasonic-assisted Micro-grinding with Electroplated Diamond Wheels, J. Jpn. Soc. Grind. Eng., Vol. 53, No. 1, pp.45-48.

[2] Shimada, K., Tateishi, T., Yoshihara, N., Yan, J. and Kuriyagawa, T., 2010, Ultrasonic-assisted Micro-grinding Using Electroplated Diamond
Wheels : $2^{\text {nd }}$ Report : Effect of Ultrasonic Vibration on Workpiece Removal in Grinding with Wheel End, J. Jpn. Soc. Grind. Eng., Vol. 54, No. 1, pp.37-40.

[3] Kumabe, J., 1961, Ultrasonic Grinding J.Jpn. Soc. Prec. Eng. Vol. 27, No. 6, pp369-371 (In Japanese).

[4] Nomura, M., Wu, Y., Kato, M. and Kuriyagawa, T., 2005, Effects of Ultrasonic Vibration in Truing and Dressing of CBN Grinding Wheel Used for Internal Grinding of Small Holes, Key Engineering Materials, Vol. 291-292, pp. 183-188.

[5] Tanaka, Y., Tsuwa, H. and Yano, A.,1964, Study on the Fine Finishing by an Ultrasonically Vibrated Abrasive Stone (2nd Report) : On the Fine Finishing Resistance J. Jpn.Soc. Prec. Eng. Vol. 30, No. 3, pp. 253-258 (In Japanese).

[6] Unno, K., Nishimura, K., Minagawa, A. and Tomimori, H., 1986, The Trial Construction of Ultrasonic Grinding Equipment and the Machining Characteristics : Study on High Efficient Grinding for Brittle Materials (1st Report), J. Jpn. Soc. Prec. Eng. Vol. 52 No. 1, (1986), pp.107-113 (In Japanese).

[7] Nakagawa, H., Hirogaki, T., Iwasaki, Y., Hayashi, T., Kita, Y. and Kakino, Y., 2002, Study on Oscillation Grinding by Numerical Control with a Machining Center, J. Jpn. Soc. Prec. Eng. Vol. 68 No. 7, pp.923-927 (In Japanese).

[8] Yasui, H., and Yamazaki, G., 2003, Possibility of Ultra-Smoothness Grinding of Fine Ceramics Using a Coarse Grain Size Diamond Wheel, J. Jpn. Soc. Prec. Eng. Vol. 69 No. 1, pp.115-119 (In Japanese).

[9] Ono, K., 1952, Studies on Grinding (On the Roughness of Finished Surface), J. Jpn. Soc. Mech. Eng. Vol. 18, No. 74, pp.21-26 (In Japanese).

[10] Sato, K., 1950, Theory of grinding (Part2) J. Jpn. Soc. Prec. Eng. Vol. 16, No. 187, pp.117-123 (In Japanese).

[11] Orioka, T., 1960, Theory of Probability on Generating Process of Finished Surface in Grinding Operation J. Jpn. Soc. Mech. Eng. Vol. 63, No. 499, pp. 1185-1193 (In Japanese).

[12] Matsui, S., 1980, Statistical Approach to Grinding Mechanism : Theoretical Analysis in the Case where the Value of $\mathrm{v} / \mathrm{V}$ is not negligible as compared with the Value 1, J. Jpn. Soc. Prec. Eng. Vol. 46, No. 3, (1980), pp.298-304 (In Japanese).

[13] Matsui, S. and Syoji, K., 1970, A Statistical Approach to Grinding Mechanism: Undeformed Chip Lhip Length in Grinding J. Jpn. Soc. Prec. Eng. Vol. 36, No. 421, pp.115-120 (In Japanese).

[14] Matsui, S. and Syoji, K., 1970, Statistical Approach to Grinding Mechanism (2nd Report) : Grain Depth of Cut in Grinding, J. Jpn. Soc. Prec. Eng. Vol. 36, No. 422, pp.196-201 (In Japanese).

[15] Matsui, S. and Syoji, K., 1971, Statistical Approach to Grinding Mechanism (3rd Report) : On a Few Experiments, J. Jpn. Soc. Prec. Eng. Vol. 37, No. 441, pp.708-714 (In Japanese).

[16] Matsui, S. and Syoji, K., 1968, A Study on Effective Cutting Edges in Grinding Wheel J. Jpn. Soc. Prec. Eng. Vol. 34, No. 406, pp.743-748 (In Japanese). 